\title{
Mixed Nervous System Involvement in a Child with Multisystem Inflammatory Syndrome- A Rare Presentation of SARS-CoV-2
}

\author{
Aysha Fakhroo ${ }^{1}$, Naveen Divakaran², Fareedul Hassan², Gabriel Fox ${ }^{3}$, Imelda Lambert ${ }^{3}$ and Ahood \\ Almuslamani ${ }^{4}$
}

${ }^{1}$ Senior House Officer, Department of Pediatrics, King Hamad University Hospital, Kingdom of Bahrain ${ }^{2}$ Specialist Registrar, Department of Pediatrics, King Hamad University Hospital, Kingdom of Bahrain

${ }^{3}$ Consultant in Pediatrics, Department of Pediatrics, King Hamad University Hospital, Kingdom of Bahrain

${ }^{4}$ Consultant in Pediatric Neurology, Department of Pediatrics, King Hamad University Hospital, Kingdom of Bahrain

\begin{abstract}
Multi-system inflammatory syndrome (MIS-C) is a rare but severe complication of Covid-19 infection. The clinical presentation ranges from a mild course of systemic inflammatory response to a life threatening hyper inflammatory shock and multi organ dysfunction. Central nervous system presentation is seen in acute Covid-19 infection in adults but is rarely seen in children. We describe a case of MIS-C in a child who was admitted to our Pediatric Intensive Care Unit with features of hyper inflammatory syndrome and mixed central and peripheral nervous system manifestations.
\end{abstract}

Keywords

MIS-C, COVID-19, Hyper inflammatory syndrome, Kawasaki disease, SARS-COV-2

\section{Introduction}

Multi-system inflammatory syndrome in children (MIS-C) is a new life-threatening condition that is temporally associated with SARS Coronavirus (Covid-19). The cases occurr in children who test positive for current or recent infection by SARS-CoV-2, based on reverse-transcriptase polymerase chain reaction (RT-PCR) or serologic assay. Patients present with a persistent fever of $>3$ days duration and a constellation of symptoms including rash, bilateral non-purulent conjunctivitis or mucocutaneous inflammation, hypotension or shock, multi organ (e.g., cardiac, gastrointestinal, renal, hematologic, dermatologic and neurologic) involvement and elevated inflammatory markers with an evidence of COVID-19 infection in the absence of other causes of systemic inflammatory response [1]. It has been suggested that the syndrome results from an abnormal immune response to the virus, with clinical similarities to Kawasaki disease (KD), macrophage activation syndrome (MAS), and cytokine release syndrome [2]. However, based on the available studies, MIS-C appears to have an immunophenotype that is distinct from KD and MAS $[3,4]$.

Over 700 cases in the pediatric population were reported worldwide from March 2020 to date with clinical presentations varying from an uncomplicated Kawasaki like syndrome to life threatening hyper inflammatory shock and multi organ dysfunction. The angiotensin-converting enzyme 2 (ACE2), expressed in both neurons and glial cells is the main hostcell receptor of SARS-CoV-2, responsible for the neurological manifestations in cases of CNS dysfunction in patients affected by COVID-19 infections [5]. We report a 10-years-old girl with MIS-C who presented with a rare neurological manifestation involving both the central and the peripheral nervous system.

\section{Case Description}

In late August 2020, a previously healthy 10 -years-old girl attended the emergency department with a 5 day history of

*Corresponding author: Aysha Fakhro, Senior House Officer, Department of Pediatrics, King Hamad University Hospital, Kingdom of Bahrain

Accepted: June 07, 2021

Published online: June 09, 2021

Citation: Fakhroo A, Divakaran N, Hassan F, et al. (2021) Mixed Nervous System Involvement in a Child with Multisystem Inflammatory Syndrome- A Rare Presentation of SARS-CoV-2. J Pediatr Neurol Neurosci 5(1):119-124 
Citation: Fakhroo A, Divakaran N, Hassan F, et al. (2021) Mixed Nervous System Involvement in a Child with Multisystem Inflammatory Syndrome- A Rare Presentation of SARS-CoV-2. J Pediatr Neurol Neurosci 5(1):119-124

high-grade fever $\left(39^{\circ} \mathrm{C}\right)$, cough, generalized weakness and reduced activity. On clinical examination she was found to have non-purulent bilateral conjunctival injection and generalized erythematous rash. She was inattentive, confused with incomprehensible speech and depressed mood. Cranial nerve examination was normal. She was unable to walk with generalized hypotonia, and grade -3 power in the distal and proximal muscle groups in both upper and lower limbs. Her reflexes were intact initially with equivocal plantar response without signs of in coordination. She had nuchal rigidity with negative Kernig's and Brudzinski signs. She developed persistent tachypnoea of $40 /$ min with reduced air entry more on the left side after 2 days. Abdominal examination showed enlarged soft liver of $2 \mathrm{~cm}$ with rounded edge below the costal margin and a span of $11 \mathrm{~cm}$. Cardiac examination was normal. Echocardiography did not reveal any myocardial or coronary involvement.

In view of evolving encephalopathy, muscle weakness she was admitted to the PICU. She became more irritable and developed visual hallucination with ascending hyporeflexia evolving to areflexia over a period of 72 hours.

Laboratory investigations (Table 1) showed anemia, thrombocytopenia, normal leukocyte count (with differential neutronphilia, raised pro inflammatory markers (ESR, LDH, Ferritin, IL6), and mildly elevated liver enzymes, hypoalbuminemia with increased Fibrinogen and D-dimer. The Covid serology was strongly positive. CSF biochemistry showed (RBC 50 cells $/ \mathrm{mm}^{3}$, WBC 0 cells $/ \mathrm{mm}^{3}$, Glucose $3.7 \mathrm{mmol} / \mathrm{L}$, Protein $16.6 \mathrm{mg} / \mathrm{dL}$ ) which was within normal limits. Menin-

Table 1: Laboratory investigations.

\begin{tabular}{|l|l|l|}
\hline Lab & Result & $\begin{array}{l}\text { Normal reference } \\
\text { range }\end{array}$ \\
\hline Hemoglobin & $8.4 \mathrm{~g} / \mathrm{dL}$ & $11-15.5 \mathrm{~g} / \mathrm{dL}$ \\
\hline White blood cell & $6.92 \times 10^{9} / \mathrm{L}$ & $4-11 \times 10^{9} / \mathrm{L}$ \\
\hline Neutrophils percentage & $87 \%$ & $40-60 \%$ \\
\hline Platelets & $93 \times 10^{9} / \mathrm{L}$ & $150-450 \times 10^{9} / \mathrm{L}$ \\
\hline CRP & $\mathrm{CRP} 162 \mathrm{mg} / \mathrm{L}$ & $0-3 \mathrm{mg} / \mathrm{L}$ \\
\hline ESR & $53 \mathrm{~mm} / \mathrm{hr}$ & $0-30 \mathrm{~mm} / \mathrm{hr}$ \\
\hline LDH & $528 \mathrm{U} / \mathrm{L}$ & $100-300 \mathrm{U} / \mathrm{L}$ \\
\hline Ferritin & $5736 \mathrm{ng} / \mathrm{ml}$ & $7-140 \mathrm{ng} / \mathrm{ml}$ \\
\hline IL-6 & $82 \mathrm{pg} / \mathrm{ml}$ & $0-4.4 \mathrm{pg} / \mathrm{mL}$ \\
\hline Bilirubin total & $14 \mathrm{umol} / \mathrm{L}$ & $<26 \mathrm{umol} / \mathrm{L}$ \\
\hline Bilirubin direct & $8.48 \mathrm{umol} / \mathrm{L}$ & $0-5 \mathrm{umol} / \mathrm{L}$ \\
\hline Alanine aminotransferase & $105 \mathrm{U} / \mathrm{L}$ & $16-63 \mathrm{U} / \mathrm{L}$ \\
\hline $\begin{array}{l}\text { Gamma Glutamyl } \\
\text { transferase }\end{array}$ & $67 \mathrm{U} / \mathrm{L}$ & $<35 \mathrm{U} / \mathrm{L}$ \\
\hline $\begin{array}{l}\text { Aspartate } \\
\text { Aminotransferase }\end{array}$ & $90 \mathrm{U} / \mathrm{L}$ & $15-41 \mathrm{U} / \mathrm{L}$ \\
\hline Albumin & $18 \mathrm{~g} / \mathrm{L}$ & $30-50 \mathrm{~g} / \mathrm{L}$ \\
\hline Fibrinogen & $403 \mathrm{mg} / \mathrm{dl}$ & $217-496 \mathrm{mg} / \mathrm{dL}$ \\
\hline D-Dimer & $13 \mathrm{mg} / \mathrm{L}$ & $<0.50 \mathrm{mg} / \mathrm{L}$ \\
\hline Covid serology & $97 \mathrm{AU} / \mathrm{ml}$ & $<1.0 \mathrm{AU} / \mathrm{ml}$ \\
\hline & & \\
\hline
\end{tabular}

gitis panel was negative for neurotropic viruses and bacteria (E. coli K1, Streptococcus agalactiae, Streptococcus pneumonia, Enterovirus, Herpes simplex virus 1, Herpes simplex virus 2, Human herpes virus 6, Human parechovirus, Varicella zoster virus, Cryptococcus neoformans/gattii, Hemophilus influenza, Listeria monocytogenes, Neisseria meningitidis and cytomegalovirus). CSF culture was negative. Other investigations included negative CSF oligoclonal band, antiganglioside antibody profile in serum including GM1, GM2, GD1a, GD1b and GQ1b (Bioscientia Institute, Germany) were negative. Viral and bacterial panel in the serum (included Epstein - Barr virus, Adenovirus, Cytomegalovirus, Parvovirus, Enterovirus, Herpes simplex virus, Chlamydia and Mycoplasma pneumonia) showed no evidence of recent or past infection.

Chest X-ray done showed small left sided pleural effusion (Figure 1). MRI brain showed swelling of the splenium of the corpus callosum, with $\mathrm{T} 2$ high signal intensity and restricted diffusion (Figure 2A and Figure $2 \mathrm{~B}$ ) and MRI spine showed abnormal contrast enhancement at cauda equina leaflets at conus medullar is level measuring $1.5 \mathrm{~cm}$ (Figure $3 \mathrm{~A}$ ) revealing a mixed central and peripheral nervous system involvement. Nerve conduction study (Figure 4A and Figure 4B) showed evidence of demyelination with conduction block at the peroneal nerves, worse in the right side, wherein the right common peroneal nerve showed reduced amplitude $(1.3 \mathrm{mV})$ at the ankle and $(1.1 \mathrm{mV})$ at the fibular head (Figure $4 \mathrm{~A})$, while the left common peroneal nerve showed reduced amplitude at the ankle $(1.5 \mathrm{mV})$ and $(1.2 \mathrm{mV})$ at the fibular head (Figure $4 B)$. There was no sensory nerves involvement. Initial EEG showed generalized slowing (Figure 5).

Treatment plan was initiated as per the current MIS-C guidelines [6-8] with IVIG $2 \mathrm{~g} / \mathrm{kg}$, Aspirin and IV Methyl prednisolone $2 \mathrm{mg} / \mathrm{kg}$. She also required noninvasive ventilation for three days due to the respiratory distress. Extensive rehabilitation was started in the PICU.

Considering the clinical course and supporting investigations, a final diagnosis of MIS-C with mixed central and peripheral nervous system involvement was made. The patient improved clinically from the $4^{\text {th }}$ day of PICU admission and was fully oriented with normal speech and affect. She regained full power and normal deep tendon jerks in all limbs by the $8^{\text {th }}$ day of her PICU stay. She was supported with good nutrition and physiotherapy and was discharged from the PICU on the $9^{\text {th }}$ day after complete neurological recovery. Neuroimaging, NCV and EEG were repeated a month after discharge, MRI brain showed resolution of the abnormal signal intensity seen at the splenium of the corpus callosum (Figure 2C) MRI spine showed total resolution of contrast enhancement pattern of cauda equina leaflets at conus medullar is level (Figure 3B). Repeated nerve conduction study revealed persisting mild left sided peroneal motor neuropathy with amplitude (1.6 $\mathrm{mV})$ at the left ankle and $(1.4 \mathrm{mV})$ at the left fibular head (Figure 4C and Figure 4D), while the right common peroneal nerve showed amplitude $(2.2 \mathrm{mV})$ at the ankle and $(2.1 \mathrm{mV})$ at the fibular head (Figure 4D). EEG was also repeated and it showed normal awake and sleep pattern. 


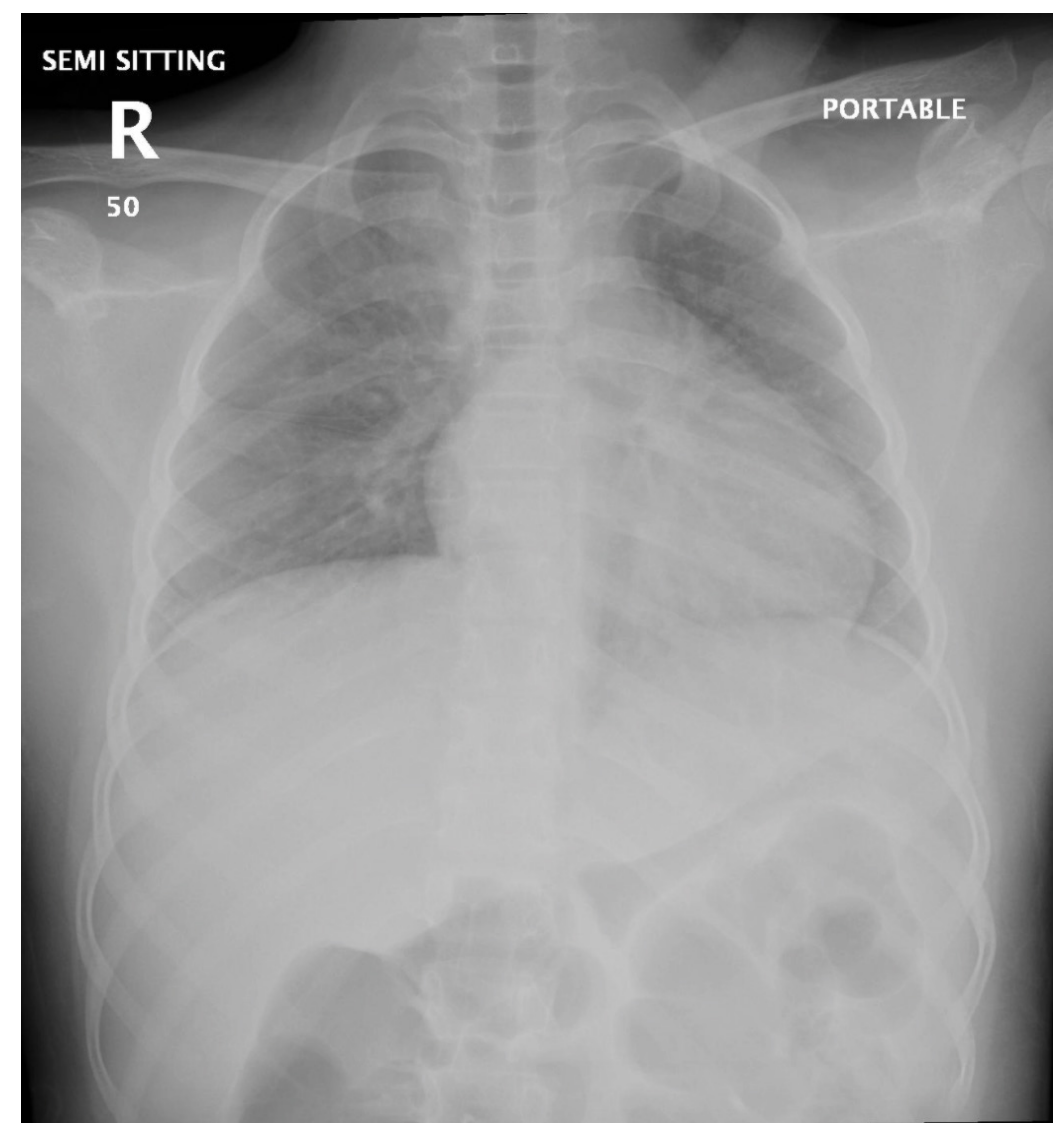

Figure 1: Chest X-ray. Showing left sided pleural effusion.
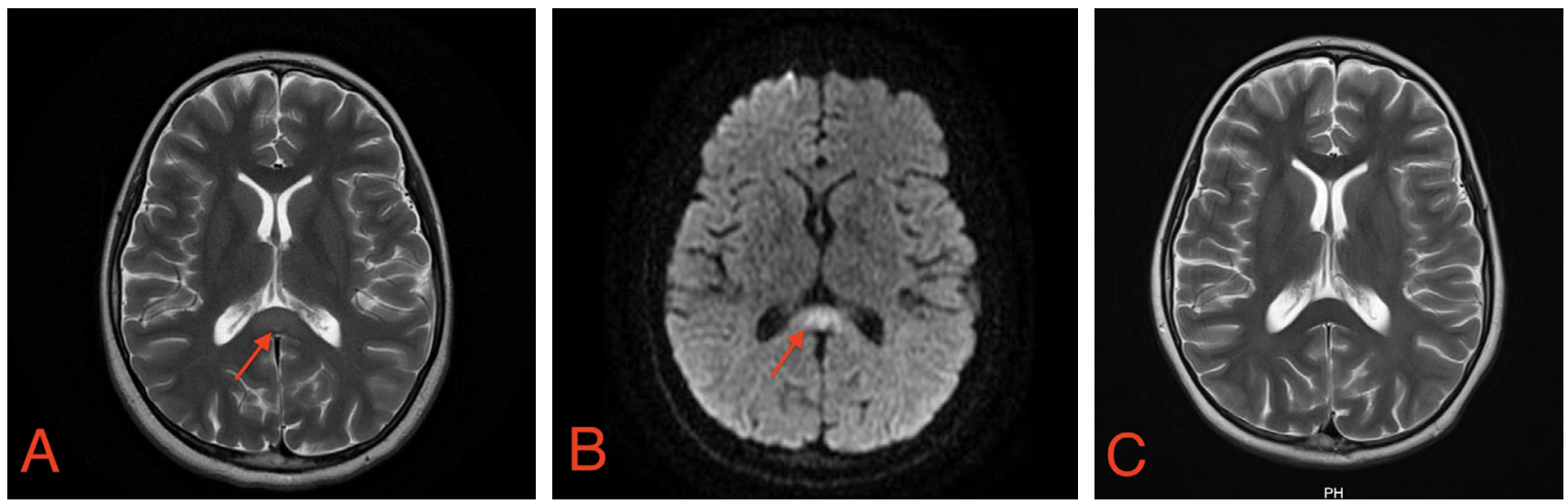

Figure 2: MRI brain (A) Axial T2; (B) Axial DWI sections showed Oedematous swollen splenium of corpus callosum showing T2 high signal intensity and restricted diffusion, findings are suggestive of transient lesions of the splenium of the corpus callosum; (C) Resolution of the abnormal signal intensity seen at the splenium of the corpus callosum.

\section{Discussion}

Our patient presented with a distinct neurological syndrome post Covid-19 associated with lesions confirmed with neuroimaging. Among the 700 reported cases to date, Abdel-Mannan, et al. [9] published a case series of 4 patients from Great Ormond Street Hospital with the similar CNS manifestation. However, the key observation in our case is the changes in the splenial corpus callosum changes along with the cauda equina changes. The former report also showed a similar splenial changes in all patients but MRI spine was not done for those patients. The systemic inflammatory response with neurological and other organ involvement shows a clinical crossway with Kawasaki disease and macrophage activation syndrome [10]. The neurological symptoms may have been part of the hyper inflammatory nature of the disease in keeping with the raised systemic inflammatory markers seen in our case. A plausible mechanism would be exposure of the immune system to new CNS antigens as a result of blood brain barrier damage from SARS-CoV-2, which causes endo- 
Citation: Fakhroo A, Divakaran N, Hassan F, et al. (2021) Mixed Nervous System Involvement in a Child with Multisystem Inflammatory Syndrome- A Rare Presentation of SARS-CoV-2. J Pediatr Neurol Neurosci 5(1):119-124
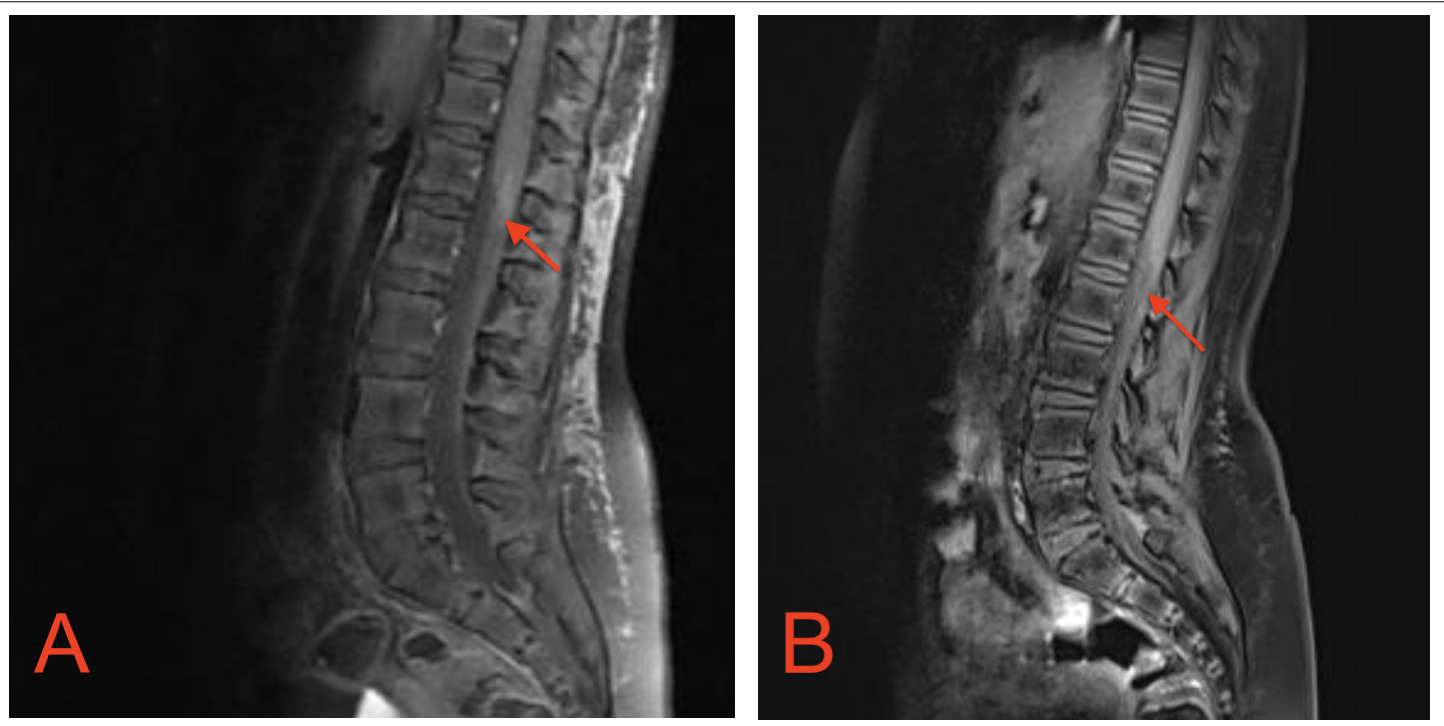

Figure 3: MRI spine (A) Abnormal contrast enhancement is noted at cauda equina leaflets at conus medullaris level for $1.5 \mathrm{~cm}$ segment; (B) Total resolution of contrast enhancement pattern of cauda equina leaflets at conus medullaris level.

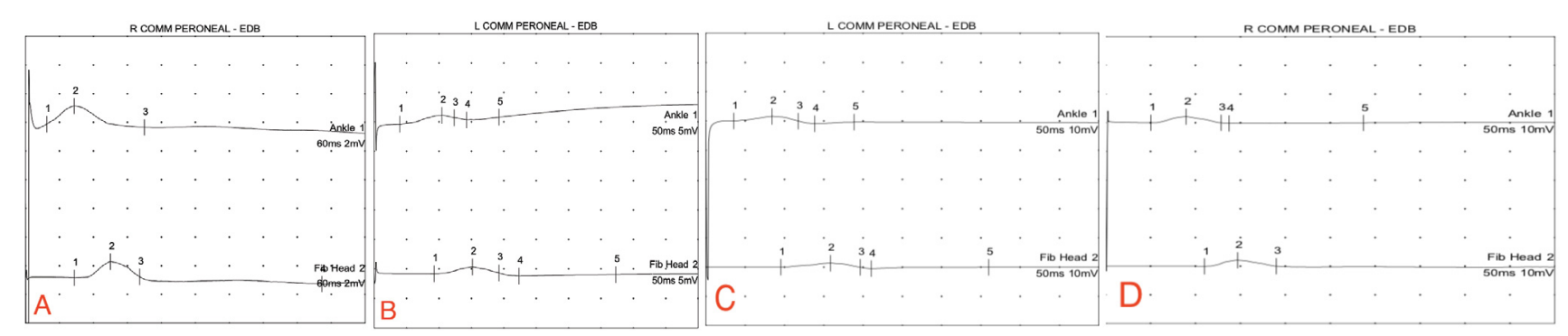

Figure 4: $(A$ and $B)$ Nerve conduction study showed evidence of demyelination (A) Right common peroneal nerve reduced amplitude $1.3 \mathrm{mV}$ at the ankle and $1.1 \mathrm{mV}$ at the fibular head; (B) Left common peroneal reduced amplitude $1.5 \mathrm{mV}$ noted at the ankle and $1.2 \mathrm{mV}$ at the fibular head; ( $C$ and D) Repeated nerve conduction study revealed persisting (C) mild left sided peroneal motor neuropathy with amplitude $(1.6 \mathrm{mV})$ at the left ankle and $(1.4 \mathrm{mV})$ at the left fibular head, while the (D) right common peroneal nerve showed amplitude $(2.2 \mathrm{mV})$ at the ankle and $(2.1 \mathrm{mV})$ at the fibular head.

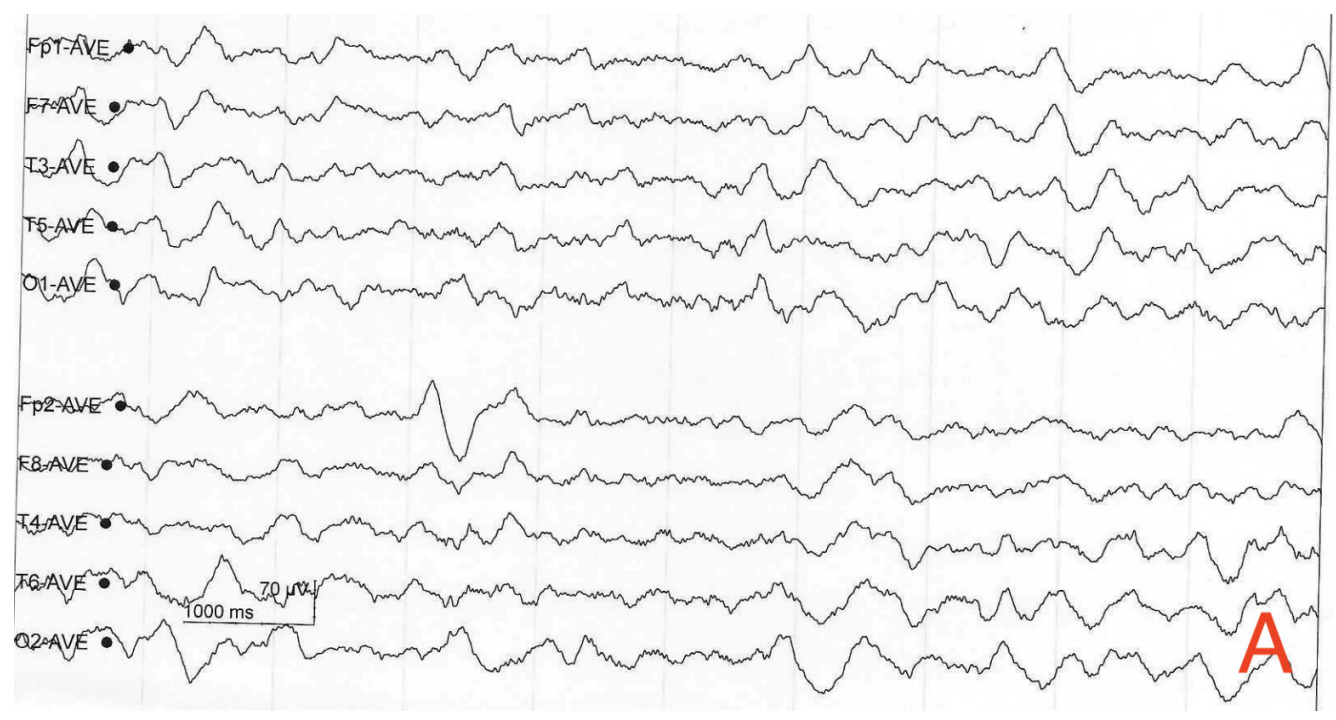

Figure 5: Electroencephalogram. Showing generalized slowing. 
Citation: Fakhroo A, Divakaran N, Hassan F, et al. (2021) Mixed Nervous System Involvement in a Child with Multisystem Inflammatory Syndrome- A Rare Presentation of SARS-CoV-2. J Pediatr Neurol Neurosci 5(1):119-124

theliopathy [11] and leads to an immune directed attack on the CNS. In studies done by Asadi Pooya, et al. and Roman, et al. $[12,13]$ compared to adult patients, children accounted for only $1-5 \%$ of COVID-19 (SARS-CoV-2) cases, of which more than $80 \%$ were asymptomatic or mild cases. SARS-CoV-2 may have neuroinvasive potential because $36 \%$ of adult patients are reported to have a variety of neurological manifestations, including headache, dizziness, acute cerebrovascular events, and changes in mental status. However, another study of 171 Chinese children with Covid-19 infection did not report neurological involvement [14]. Non-specific headaches were the only reported neurological symptoms, accounting for $4-28 \%$ of Covid-19 infected children [15].

Reviewing 187 children from the six latest reports of MIS-C cases, found an unexpectedly high incidence (34\%) of neurological involvement [16]. Compared with Kawasaki disease shock syndrome (KDSS), which shares several clinical features and severity with MIS-C, the neurological manifestations of KDSS have been found in as high as $54 \%$ of the affected children [17]. In an adult cohort in Wuhan, China [18], 78 of 214 patients (36.4\%) had neurological manifestations, which included dizziness $(n=36)$, headache $(n=28)$, impaired consciousness ( $n=16)$, acute cerebrovascular disease $(n=6)$, ataxia $(n=1)$, and seizures $(n=1)$. In comparison with patients with active non-severe infection, those with severe infection had more neurological presentations acute cerebrovascular diseases (5 [5.7\%] vs. 1 [0.8\%]) and impaired consciousness (13 [14.8\%] vs. 3 [2.4\%]). Neuroimaging, cerebrospinal fluid, and neurophysiology tests were not performed in this cohort.

A key observation in our patient was the acute splenial lesion seen on brain MRI and cauda equina lesion on spine MRI. Interestingly, a typical, transient, oval-shaped lesion in the median aspect of the splenium of the corpus callosum, either in isolation or with more extensive brain involvement, has also been reported [19] in children with Kawasaki disease. The exact function of the splenium is not completely understood, but a splenial lesion may result in the disconnection of the cerebral hemispheres, with disruption of higher cortical function, loss of conscious processes and delirious behavior [20]. General differential diagnosis of lesions involving the splenium includes ischemia, infections, posterior reversible encephalopathy syndrome, diffuse axonal injury, multiple sclerosis, hydrocephalus, adrenoleukodystrophy, lymphoma, epilepsy etc [21]. The abnormal enhancement in the cauda equine carries a broad differential diagnosis, the commonest being post-infectious inflammatory condition like Guillain Barre syndrome, spinal meningitis and less commonly vasculitis neuropathies [22].

Considering the course of the illness and full recovery following IVIG and Methylprednisolone, negative antiganglioside antibody profile, with the characteristic MRI spine and Nerve Conduction Velocity test findings, a diagnosis of Multi-system inflammatory syndrome (MIS-C) post Covid-19 infection presenting with encephalomyelitis and Guillain Barre like acute inflammatory demyelinating polyradiculoneuropathy was made. Her neurological symptoms could have been part of the hyper inflammatory syndrome in keeping with the raised systemic inflammatory markers.

\section{Conclusion}

We report a case of a 10-year-old girl with COVID-19 who had a clinical phenotype involving both the central and the peripheral nervous system. The symptoms developed after 4 weeks of the girl testing positive for Covid RT PCR. The normal CSF study, rapid response to immunosuppression, and the clinical overlap with Kawasaki like and macrophage activation syndrome suggest that this is likely to be an immune mediated. Clinicians should consider SARS-CoV-2 in their differential diagnosis for children presenting with acute onset neurological symptoms particularly with these imaging findings while still exploring other possible causes.

In conclusion, further investigations on the pediatric COVID-19 population, including the brain MRI examination, CSF study, and children-specific therapies, are encouraged to recognize this emerging neurological complication. Clinician should have a high index of suspicion and close neurological surveillance is required to assess the neurological outcomes in these patients.

\section{References}

1. World Health Organization (2020) Multisystem inflammatory syndrome in children and adolescents with COVID-19.

2. Garcia-Pavon S, Yamazaki-Nakashimada MA, Baez M, et al. (2017) Kawasaki disease complicated with macrophage activation syndrome: A systematic review. J Pediatr Hematol Oncol 39: 445-451.

3. Carter MJ, Fish M, Jennings A, et al. (2020) Peripheral immunophenotypes in children with multisystem inflammatory syndrome associated with SARS-CoV-2 infection. Nat Med 26: 1701-1707.

4. Lee PY, Day-Lewis M, Henderson LA, et al. (2020) Distinct clinical and immunological features of SARS-CoV-2-induced multisystem inflammatory syndrome in children. J Clin Invest 130: 5942-5950.

5. BaigAM, Khaleeq A, Ali U, et al. (2020) Evidence of the COVID-19 virus targeting the CNS: Tissue distribution, host-virus interaction, and proposed neurotropic mechanisms. ACS Chem Neurosci 11: 995-998.

6. McCrindle BW, Rowley AH, Newburger JW, et al. (2017) Diagnosis, Treatment, and Long-Term Management of Kawasaki Disease: A Scientific Statement for Health Professionals from the American Heart Association. Circulation 135: e927.

7. American Academy of Pediatrics (2018) Kawasaki disease. In: Kimberlin DW, Brady MT, Jackson MA, et al., Red Book: 2018 Report of the Committee on Infectious Diseases. ( $31^{\text {st }}$ edn), American Academy of Pediatrics. Itasca, IL, USA, 490.

8. Kobayashi T, Saji T, Otani T, et al. (2012) Efficacy of immunoglobulin plus prednisolone for prevention of coronary artery abnormalities in severe Kawasaki disease (RAISE study): A randomised, open-label, blinded-endpoints trial. Lancet 379: 1613-1620.

9. Abdel-Mannan O, Eyre M, Löbel U, et al. (2020) Neurologic and Radiographic Findings Associated With COVID-19 Infection in Children. JAMA Neurol 77: 1440-1445.

10. Henter JI, Elinder G, Söder O, et al. (1991) Incidence in Sweden and clinical features of familial hemophagocytic lymphohistiocytosis. Acta Paediatr Scand 80: 428-435.

11. Varga Z, Flammer AJ, Steiger $P$, et al. (2020) Endothelial cell infection and endotheliitis in COVID-19. Lancet 395: 1417-1418. 
Citation: Fakhroo A, Divakaran N, Hassan F, et al. (2021) Mixed Nervous System Involvement in a Child with Multisystem Inflammatory Syndrome- A Rare Presentation of SARS-CoV-2. J Pediatr Neurol Neurosci 5(1):119-124

12. Asadi-Pooya AA, Simani L (2020) Central nervous system manifestations of COVID-19: A systematic review. J Neurol Sci 413: 116832.

13. Roman GC, Spencer PS, Reis J, et al. (2020) The neurology of COVID-19 revisited: A proposal from the environmental neurology specialty group of the world federation of neurology to implement international neurological registries. J Neurol Sci 414: 116884.

14. X Lu, L Zhang, H Du, et al. (2020) Chinese pediatric novel coronavirus study, SARS-CoV-2 infection in children. N Engl J Med 382: 1663-1665.

15. Parri M, Lenge D, Buonsenso $G$ (2020) Coronavirus infection in pediatric emergency departments research: Children with Covid-19 in pediatric emergency departments in italy. N Engl J Med 383: 187-190.

16. Z Belhadjer, M Meot, F Bajolle, et al. (2020) Acute heart failure in multisystem inflammatory syndrome in children (MIS-C) in the context of global SARS-CoV-2 pandemic Circulation.
17. Gamez-Gonzalez LB, Moribe-Quintero I, Cisneros-Castolo M, et al. (2018) Kawasaki disease shock syndrome: Unique and severe subtype of Kawasaki disease. Pediatr Int 60: 781-790.

18. Mao L, Jin H, Wang M, et al. (2020) Neurologic manifestations of hospitalized patients with coronavirus disease 2019 in Wuhan, China. JAMA Neurol 77: 683-690.

19. Kontzialis M, Soares BP, Huisman TAGM (2017) Lesions in the splenium of the corpus callosum on MRI in children: $\mathrm{S}$ review. J Neuroimaging 27: 549-561.

20. Takanashi J, Tada H, Kuroki H, et al. (2009) Delirious behavior in influenza is associated with a reversible splenial lesion. Brain Dev 31: 423-426.

21. Horne A, Trottestam H, Aricò M, et al. (2008) Frequency and spectrum of central nervous system involvement in 193 children with haemophagocytic lymphohistiocytosis. Br J Haematol 140: 327-335.

22. Ross JS (2005) Diagnostic Imaigng: Spine. ( $1^{\text {st }}$ edn), Salt Lake City: Amirsys, USA. 